\title{
The automatic alignment system of GEO 600
}

\author{
H Grote ${ }^{1,4}$, G Heinzel ${ }^{2}$, A Freise ${ }^{1}$, S Gossler ${ }^{1}$, B Willke ${ }^{1,2}$, H Lück ${ }^{1,2}$,

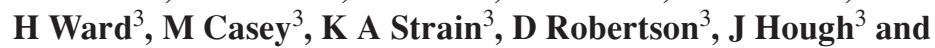 \\ K Danzmann ${ }^{1,2}$ \\ ${ }^{1}$ Institut für Atom- und Molekülphysik, Universität Hannover, Callinstrasse 38, D-30167 \\ Hannover, Germany \\ 2 Albert Einstein Institut für Gravitationsphysik, Aussenstelle Hannover, Callinstrasse 38, \\ D-30167 Hannover, Germany \\ ${ }^{3}$ Department of Physics and Astronomy, University of Glasgow G128QQ, UK \\ E-mail: hrg@mpq.mpg.de
}

Received 2 October 2001, in final form 29 October 2001

Published 18 March 2002

Online at stacks.iop.org/CQG/19/1849

\begin{abstract}
This paper gives an overview of the automatic mirror alignment system of the modecleaner and main interferometer of the GEO 600 gravitational wave detector. In order to achieve the required sensitivity of the detector, the eigenmodes of all optical cavities have to be aligned with respect to the incoming beams (or vice versa) and kept aligned for long measuring periods. Moreover the beam spots have to be centred on the mirrors to minimize coupling of residual angular mirror motion into changes of the optical path length. An overview of the principles and setup for the automatic alignment is given, and first results of the modecleaner and $1200 \mathrm{~m}$ cavity alignment system are presented, including the error-point spectra of mirror angular motions, which are smaller than $10^{-8} \mathrm{rad} \mathrm{Hz}^{-1 / 2}$ below $10 \mathrm{~Hz}$.
\end{abstract}

PACS numbers: 0480N, 0760L, 9555Y

(Some figures in this article are in colour only in the electronic version)

\section{Introduction}

In laser interferometric gravitational wave detectors, all cavity and beam steering mirrors within the vacuum system are suspended as pendulums for the purpose of seismic isolation. While this gives good isolation in the gravitational wave measurement band (e.g. above $40 \mathrm{~Hz}$ ), the mirror motions are increased around the pendulum's main resonances (around $1 \mathrm{~Hz}$ ) and the mirrors are subjected to enhanced drifts.

4 To whom correspondence should be addressed. 


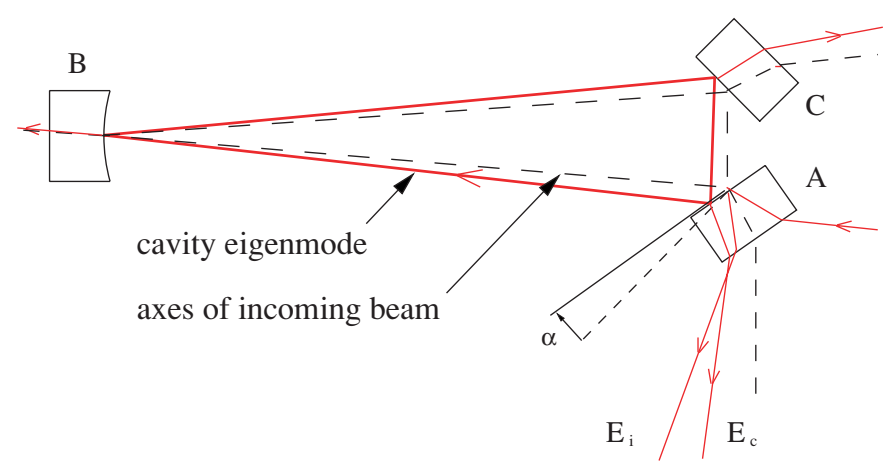

Figure 1. Schematic triangular ring cavity consisting of two flat mirrors A, C and a curved mirror B. Mirror A is misaligned by an angle $\alpha$.

To enable an optimized sensitivity as well as long-term stable operation of the detector, an automatic ${ }^{5}$ angular alignment in two degrees of freedom for each suspended mirror is required.

Within the GEO 600 [1] gravitational wave detector, there are two suspended ring-type modecleaners, the power recycling cavity and the Michelson interferometer to be aligned with such a system.

The goal of the automatic alignment system is to keep the optical eigenmode of a cavity superimposed with the axes of the respective incoming beam and centre all beam spots on the corresponding mirrors.

\section{Differential wavefront sensing}

To align a cavity's eigenmode with the incoming beam, their relative orientation has to be detected. Error signals providing this information are obtained with the differential wavefront sensing technique (see, e.g., [2]), which is similar to the Pound-Drever-Hall sensing [3].

Figure 1 shows a triangular ring cavity consisting of two flat mirrors $\mathrm{A}, \mathrm{C}$ and a curved mirror B. An incident laser light impinging on mirror A is partly reflected as an electric field $E_{\mathrm{i}}$ and partly enters the cavity. The light circulating inside the cavity leaks out at mirror $\mathrm{A}$ as field $E_{\mathrm{c}}$.

The figure shows a misalignment of mirror $\mathrm{A}$, resulting in an angle between $E_{\mathrm{i}}$ and $E_{\mathrm{c}}$. While here the misalignment is largely exaggerated, in a real situation the two beams cannot be separated, but their interference pattern can be detected with photodiodes.

Using Pound-Drever-Hall sensing, the phase difference between the wavefronts of $E_{\mathrm{i}}$ and $E_{\mathrm{c}}$ is read out over the whole cross section of the interference pattern. For this method, the incident light is being phase-modulated at an appropriate radio frequency with an electro-optic modulator (EOM). The photocurrent of a diode illuminated with $E_{\mathrm{i}}$ and $E_{\mathrm{c}}$ is then coherently demodulated. The resulting signal is a measure of the match between the incident laser frequency and one of the cavity's resonant frequencies. Usually an appropriate feedback loop is used to keep the difference between these frequencies close to zero, which is referred to as the 'locking' of a cavity.

If a cavity is locked (which is assumed for all the following discussions), the differential wavefront sensing method can measure the angle between the wavefronts of $E_{\mathrm{i}}$ and $E_{\mathrm{c}}$ by using

5 The term 'automatic' has two meanings here: a feedback system which optimizes the alignment is called 'automatic alignment'. Furthermore 'automatic alignment' describes a computer-controlled system which switches the alignment feedback loops on and off, depending on the detector status. 
a split photodiode and calculating the difference between the two demodulated photocurrents of the different photodiode sections ${ }^{6}$. With a quadrant diode this can be done for the horizontal and vertical directions simultaneously.

The angle between the wavefronts is generally not identical to the angle between the beams $E_{\mathrm{i}}$ and $E_{\mathrm{c}}$. But if the wavefront angles are measured at two different distances from the beam waist (which is located between mirrors $\mathrm{A}$ and $\mathrm{C}$ in the configuration of figure 1), four different quantities can be obtained, giving full information about angular and parallel displacement of the cavity's axes against the axis of the incoming beam.

Any other linear combination of the four detected wavefront angles may be chosen to match the coordinate system of experimentally available independent actuators, enabling feedback-controlled alignment. As a feature of the ring cavity in figure 1, any misalignment of mirror A cannot be distinguished from misalignments of mirror $\mathrm{C}$ with the described method, but both mirrors can be chosen as actuators in a common mode. Further, the linear combinations chosen for the horizontal plane are generally different from those in the vertical plane. (For details see [4].)

\section{Automatic alignment of a GEO 600 modecleaner}

Figure 2 shows the setup for the automatic alignment system of the first GEO 600 modecleaner.

The laser beam directed to mirror A is phase-modulated with a frequency of $25.2 \mathrm{MHz}$ by EOM1, enabling the use of Pound-Drever-Hall locking, and differential wavefront sensing. Steering mirror BD1 points the beam towards the cavity, constituted by the flat mirrors A and C, and curved mirror B. (For details on the GEO 600 modecleaners see [5].)

The beam consisting of the interfering fields $E_{\mathrm{i}}+E_{\mathrm{c}}$ (see figure 1 for correspondence) is split into two paths by the power beamsplitter BS1 with a reflectivity of about $50 \%$. In each path the light is directed to the quadrant diodes D1 and D2. A lens system (L1 and L2) in the light path to D2 provides a projection into the far field of $E_{\mathrm{i}}+E_{\mathrm{c}}$. With the proper adjustment of L1 and L2 the detected wavefront angles on D1 and D2 can be made as independent as possible.

The components G1 and G2 are beam pointing devices and have the purpose to centre the beams on the respective quadrant diodes D1 and D2. G1 and G2 each consist of two commercial galvanometer scanners, carrying small $(10 \times 15 \mathrm{~mm})$ mirrors on their axis that can be rotated in one degree of freedom. With a perpendicular mounting of two scanners, one unit can point a reflected beam in two degrees of freedom. The 'centring servo' loops control the spot position on the diodes with a bandwith of around $1 \mathrm{kHz}$. We use this concept to ensure that the angle between the interfering wavefronts $E_{\mathrm{i}}$ and $E_{\mathrm{c}}$ is detected correctly by the quadrant photodiodes.

If beam $E_{\mathrm{i}}+E_{\mathrm{c}}$ is not centred on the diodes, the differential wavefront signal size decreases and also includes unwanted information from higher order modes. Obviously, if beam $E_{\mathrm{i}}+$ $E_{\mathrm{c}}$ deviates from the centre of a diode by one beam radius, alignment information is almost completely lost. If $\mathrm{G} 1$ and $\mathrm{G} 2$ are not used, an angular pointing of beam $E_{\mathrm{i}}+E_{\mathrm{c}}$ of roughly $200 \mu \mathrm{rad}$ leads to such a decentring on D2. Using the scanners, beam $E_{\mathrm{i}}+E_{\mathrm{c}}$ can point up to $2000 \mu \mathrm{rad}$ while the beam is still centred on D2, yielding a correct differential wavefront sensing signal. With a larger pointing than this, the beam gets cut off at the scanner mirror edges. Given these numbers, the dynamic range of the alignment detection system is increased

6 In principle it is also possible to obtain differential wavefront signals without a locked cavity. To do this, the photodiode signals have to be read out each time the cavity is in resonance with the incident light. A computercontrolled system could provide an error signal then, which might be useful for an automated or manual coarse alignment of complex interferometers 


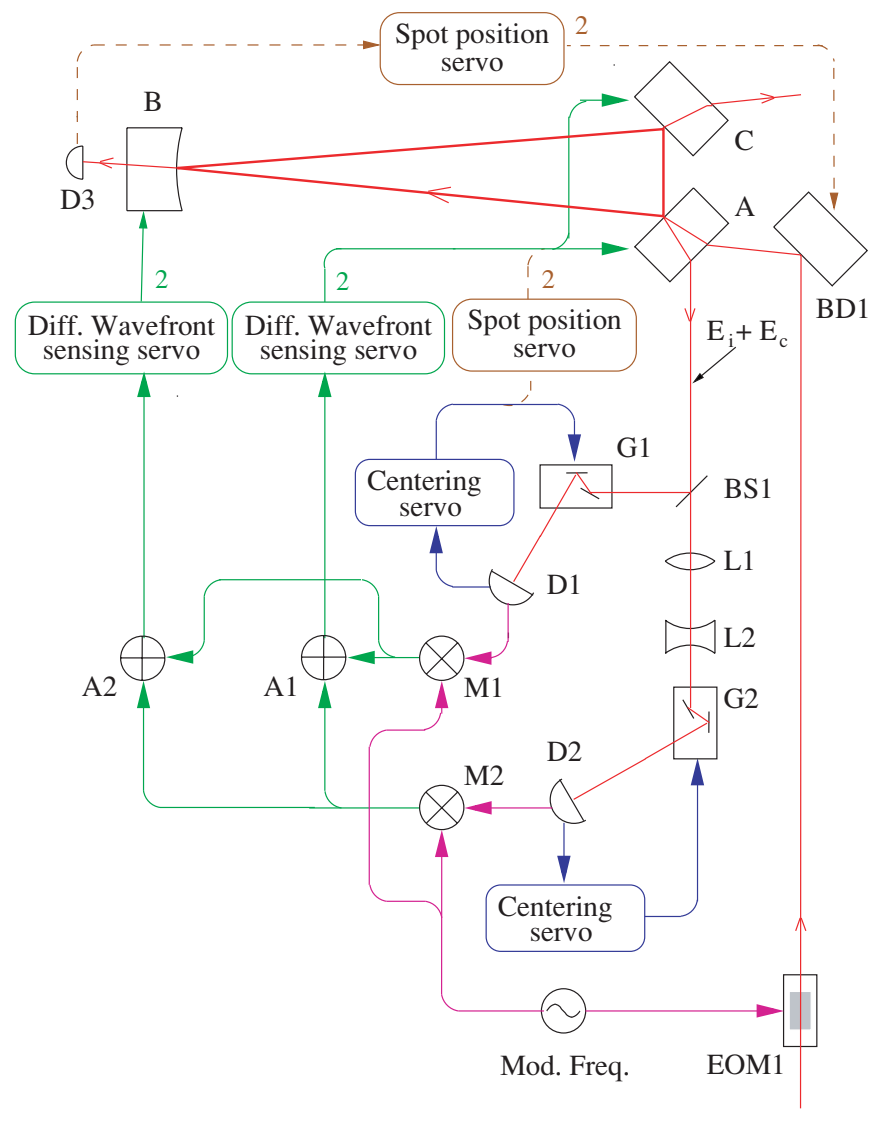

Figure 2. Automatic alignment system layout for one modecleaner. The numbers at the servo boxes count the degrees of freedom controlled by each path.

by more than a factor of 10 by the scanners. This large dynamic range allows a successful switching on of the alignment system even in largely misaligned initial states. (See figure 3 for such a condition.)

Each quadrant photodiode, D1 and D2, of the type CENTRONICS QD50-3T has a resonant electronic circuit (tuned at the modulation frequency of EOM1) for each of its quadrants to increase the signal-to-noise ratio. After demodulation, the differences between the quadrants of one photodiode give the information about the angles between the wavefronts in two degrees of freedom. Those electronics are symbolized by M1 and M2 for each photodiode.

To generate signals that correspond to the motion of individual mirrors of the ring cavity, the according linear combinations of the outputs of M1 and M2 are processed by analogue electronic circuits A1 and A2. The experimental result of this orthogonalization is better than $1 / 10$ (meaning that if, e.g., mirror B rotates out of its nominal position, this signal shows up with less than $1 / 10$ in the misalignment signal for mirrors $A$ and $C$ ).

The error signals for the mirror orientations are then amplified with the appropriate gain and frequency response and fed back to mirror $\mathrm{B}$ as well as mirrors $\mathrm{A}+\mathrm{C}$ in common mode.

As feedback actuator for the modecleaner alignment we use coils with magnets attached to the intermediate mass of each mirror's double pendulum. The transfer function from the 


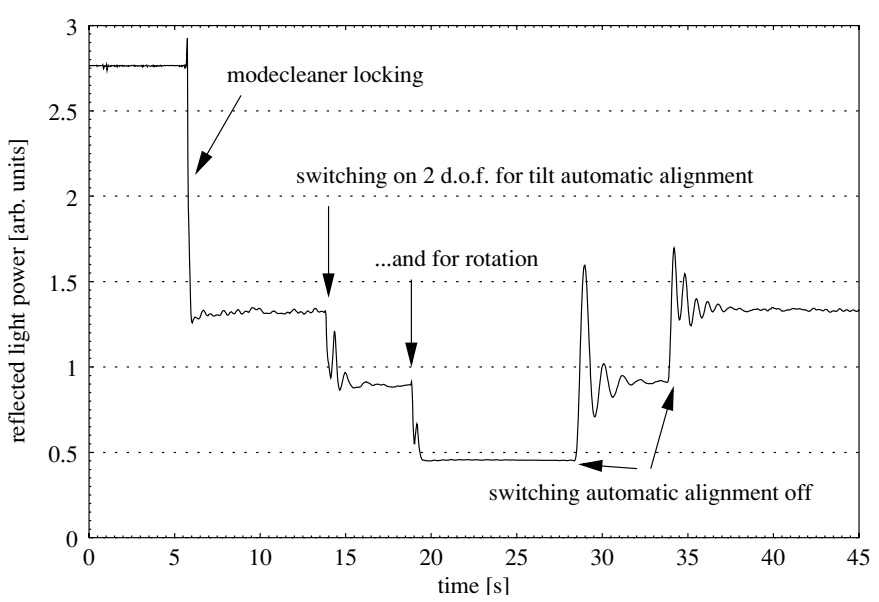

Figure 3. Switching on and off the modecleaner automatic alignment.

input of the coil drivers to the mirror's angular motion has a phase delay of $360^{\circ}$ at frequencies above the pendulum's main resonances (around $1 \mathrm{~Hz}$ ), which makes it difficult to achieve unity gain frequencies of the alignment system above $1 \mathrm{~Hz}$. Nevertheless two different feedback filters were successfully tried, one with a bandwidth of $6 \mathrm{~Hz}$ (using several differentiator stages to provide an electronic phase lead of $210^{\circ}$ around $6 \mathrm{~Hz}$ ), another one using only integrator stages yielding a bandwidth of $0.2 \mathrm{~Hz}$.

Figure 3 shows a time series of the light power reflected from the first modecleaner of GEO 600 for different operating conditions of the automatic alignment system.

Up to second 6 the cavity is unlocked and almost all light is reflected. Then the cavity is locked to an intentionally misaligned state with only around $65 \%$ of the possible power entering the cavity. At second 14 the differential wavefront control is switched on for the two tilt degrees of freedom; at second 19 feedback for the two rotational degrees of freedom is switched on. A maximum of light is now entering the cavity, limited by imperfect annular modematching at the time of measurement. It can be seen that the power fluctuations are significantly smaller than in the misaligned case, due to reduced coupling of beam geometry fluctuations. Finally the graph shows the switching off in reverse order, with the pendulums swinging back to their equilibrium positions.

We obtain information about four degrees of freedom for the cavity alignment with the differential wavefront sensing, but there are two remaining degrees of freedom of the cavity (which consists of three mirrors and thus has six angular degrees of freedom) to be controlled. These are the differential modes of mirrors $\mathrm{A}$ and $\mathrm{C}$, which can be detected by the direction of the beam $E_{\mathrm{i}}+E_{\mathrm{c}}$ propagation. As we have the quadrant photodiode D1 in place already, we can read out the spot position on the diode by reading out the quadrant photocurrents at low frequencies (in contrast to the RF readout described above). The galvanometer scanners use these signals to centre the beam on the photodiode; thus the propagation direction information of the beam $E_{\mathrm{i}}+E_{\mathrm{c}}$ is contained in the feedback signals applied to the scanners (at least within the scanners loop bandwidth of $1 \mathrm{kHz}$ ). Figure 2 shows the feedback loop using these signals which are fed back to the differential modes of mirrors $\mathrm{A}$ and $\mathrm{C}$ with a digital control loop. To avoid oscillations, this loop has to be slower than the differential wavefront sensing loop.

A similar type of feedback is the spot position control with D3, detecting the transmitted light of cavity mirror B. A digital feedback loop then aligns mirror BD1, which determines 


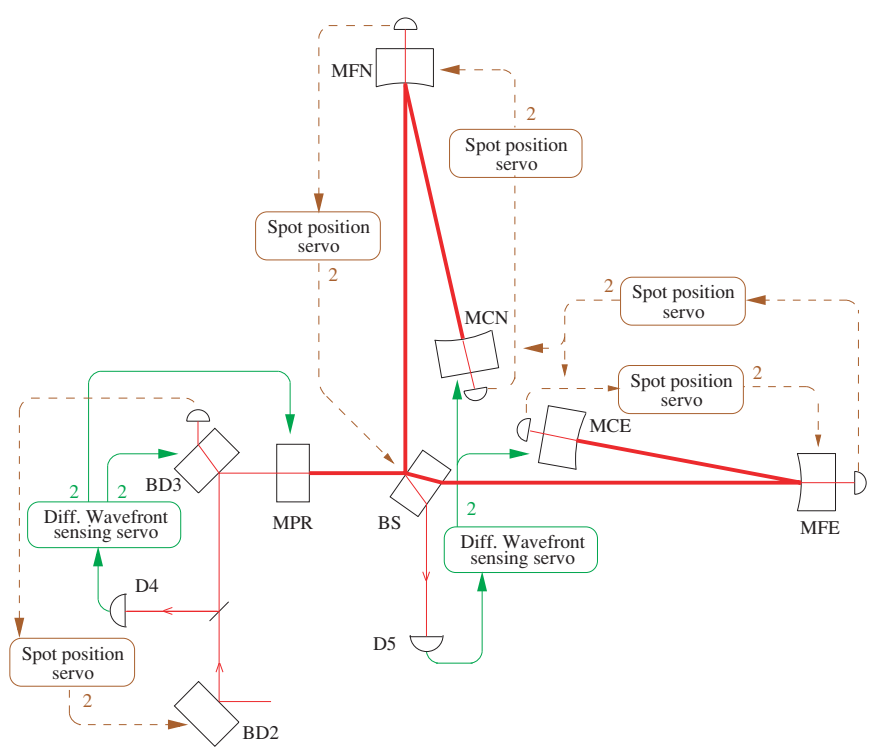

Figure 4. Automatic alignment system layout for the power-recycled Michelson interferometer.

the spot position on D3, as long as the cavity axes follow the axes of the incoming beam. Thus care has to be taken that this spot position control is only active (again with a low enough bandwidth) when the differential wavefront feedback is working.

\section{Main interferometer automatic alignment}

Figure 4 gives a brief overview of the alignment system for the power-recycled Michelson interferometer (signal recycling not included).

The light leaving the modecleaners is directed by BD2 and BD3 to the power recycling cavity. The light reflected from MPR is detected by D4, which symbolizes a differential wavefront sensing unit for four degrees of freedom like the one described for the modecleaner section. In the current configuration feedback is applied to BD3 and MPR to align the power recycling cavity's axes with the axes of the incoming beam. Both mirrors have magnets attached on which forces are applied with coils.

Alignment information for the Michelson interferometer is obtained by D5, which symbolizes a unit for differential wavefront sensing for two degrees of freedom. Feedback in this case is applied to the cavity folding mirrors $\mathrm{MCE}$ and $\mathrm{MCN}$ differentially. The common mode of these two mirrors is used for spot position control. One simplified possible topology of the spot position control for all remaining mirrors is shown, but others are possible.

Figure 5 shows the error point spectrum of the power recycling mirror's horizontal alignment degree of freedom.

At the time of this measurement in January 2001 the beamsplitter BS was not installed, and the mirrors MPR, MFE and MCE formed a $1200 \mathrm{~m}$ long folded Fabry-Perot cavity, described in [6]. The measurement shows the angular motion of mirror MPR against the incoming beam without automatic alignment and with automatic alignment for four degrees of freedom switched on. With a bandwidth of the alignment feedback loops around $10 \mathrm{~Hz}$, the rms value of 


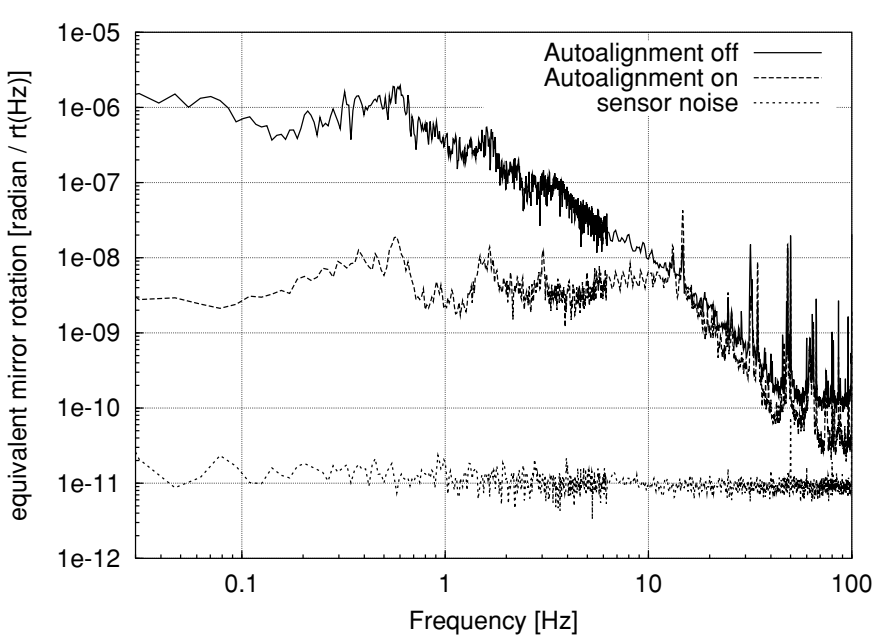

Figure 5. Horizontal alignment spectrum of mirror MPR with and without automatic alignment.

the angular motion is damped to $0.02 \mu \mathrm{rad}$ compared to $1.0 \mu \mathrm{rad}$ without automatic alignment. The electronic noise of the differential wavefront sensor is as low as $10^{-11} \mathrm{rad} \mathrm{Hz}^{-1 / 2}$.

\section{Conclusion}

The complete automatic alignment system for the two $8 \mathrm{~m}$ GEO 600 suspended ring type modecleaners is installed and has been in continuous operation since spring 2001. No manual mirror alignment has been required since then.

Furthermore, the automatic alignment of a kilometre scale interferometer with differential wavefront sensing in four degrees of freedom was successfully demonstrated as a major part of the complete automatic alignment of GEO 600.

\section{Acknowledgments}

The authors would like to thank PPARC in the UK, the BMBF and the State of Lower Saxony in Germany.

\section{References}

[1] Danzmann K et al 1994 1st Edoardo Amaldi Conf. on Gravitational Wave Experiments (Frascati 1994) (Singapore: World Scientific) pp 100-11

[2] Morrison E, Meers B J, Robertson D I and Ward H 1994 Automatic alignment of optical interferometers Appl. Opt. 335041

[3] Drever R W P, Hall J L, Kowalski F V, Hough J, Ford G M, Munley A J and Ward H 1983 Laser phase and frequency stabilisation using an optical resonator Phys. Rev. B 3197

[4] Heinzel G 1999 Advanced optical techniques for laser-interferometric gravitational-wave detectors $P h D$ Thesis University of Hannover, pp141-6 (also available as MPQ report 243)

[5] Gossler S et al 2002 The modecleaner system and suspension aspects of GEO 600 Proc. of the 4th Edoardo Amaldi Conf. on Gravitational Waves (Perth, Western Australia, 8-13 July 2001) Class. Quantum Grav. 19 1835

[6] Freise A et al 2002 Performance of a $1200 \mathrm{~m}$ long suspended Fabry-Perot cavity Proc. of the 4th Edoardo Amaldi Conf. on Gravitational Waves (Perth, Western Australia, 8-13 July 2001) Class. Quantum Grav. 191389 